\title{
Front Matter: Volume 7432
}

, "Front Matter: Volume 7432," Proc. SPIE 7432, Optical Inspection and Metrology for Non-Optics Industries, 743201 (22 September 2009); doi: 10.1117/12.837052

SPIE Event: SPIE Optical Engineering + Applications, 2009, San Diego, California, SPIE. United States 


\title{
PROCEEDINGS OF SPIE
}

\section{Optical Inspection and Metrology for Non-Optics Industries}

\author{
Peisen S. Huang \\ Toru Yoshizawa \\ Kevin G. Harding \\ Editors
}

3-4 August 2009

San Diego, California, United States

Sponsored and Published by

SPIE 
The papers included in this volume were part of the technical conference cited on the cover and title page. Papers were selected and subject to review by the editors and conference program committee. Some conference presentations may not be available for publication. The papers published in these proceedings reflect the work and thoughts of the authors and are published herein as submitted. The publisher is not responsible for the validity of the information or for any outcomes resulting from reliance thereon.

Please use the following format to cite material from this book:

Author(s), "Title of Paper," in Optical Inspection and Metrology for Non-Optics Industries, edited by Peisen S. Huang, Toru Yoshizawa, Kevin G. Harding, Proceedings of SPIE Vol. 7432 (SPIE, Bellingham, WA, 2009) Article CID Number.

ISSN 0277-786X

ISBN 9780819477224

Published by

SPIE

P.O. Box 10, Bellingham, Washington 98227-0010 USA

Telephone +1 3606763290 (Pacific Time) · Fax +1 3606471445

SPIE.org

Copyright (C) 2009, Society of Photo-Optical Instrumentation Engineers

Copying of material in this book for internal or personal use, or for the internal or personal use of specific clients, beyond the fair use provisions granted by the U.S. Copyright Law is authorized by SPIE subject to payment of copying fees. The Transactional Reporting Service base fee for this volume is $\$ 18.00$ per article (or portion thereof), which should be paid directly to the Copyright Clearance Center (CCC), 222 Rosewood Drive, Danvers, MA 01923. Payment may also be made electronically through CCC Online at copyright.com. Other copying for republication, resale, advertising or promotion, or any form of systematic or multiple reproduction of any material in this book is prohibited except with permission in writing from the publisher. The CCC fee code is 0277-786X/09/\$18.00.

Printed in the United States of America.

Publication of record for individual papers is online in the SPIE Digital Library.

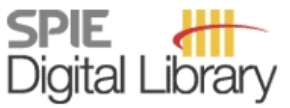

SPIEDigitalLibrary.org

Paper Numbering: Proceedings of SPIE follow an e-First publication model, with papers published first online and then in print and on CD-ROM. Papers are published as they are submitted and meet publication criteria. A unique, consistent, permanent citation identifier (CID) number is assigned to each article at the time of the first publication. Utilization of CIDs allows articles to be fully citable as soon they are published online, and connects the same identifier to all online, print, and electronic versions of the publication. SPIE uses a six-digit CID article numbering system in which:

- The first four digits correspond to the SPIE volume number.

- The last two digits indicate publication order within the volume using a Base 36 numbering system employing both numerals and letters. These two-number sets start with 00, 01, 02, 03, 04, $05,06,07,08,09,0 A, 0 B \ldots 0 Z$, followed by 10-1Z, 20-2Z, etc.

The CID number appears on each page of the manuscript. The complete citation is used on the first page, and an abbreviated version on subsequent pages. Numbers in the index correspond to the last two digits of the six-digit CID number. 


\section{Contents}

vii Conference Committee

ix Optical design dependence on technology development (Plenary Paper) [7428-01]

I. A. Neil, ScotOptix (Switzerland)

\section{CALIBRATION AND ANALYSIS METHODS I: CALIBRATION}

743202 Optimal checkerboard selection for structured light system calibration [7432-01]

W. Lohry, Y. Xu, S. Zhang, lowa State Univ. (United States)

743203 Measurement accuracy of fringe projection depending on surface normal direction [7432-02]

P. Kühmstedt, C. Bräuer-Burchardt, G. Notni, Fraunhofer IOFJena (Germany)

743204 High precision calibration method of intrinsic parameters for fish-eye cameras [7432-03] H. Komagata, Saitama Medical Univ. (Japan) and Niigata Univ. (Japan); I. Ishii, Saitama Medical Univ. (Japan); H. Makino, Niigata Univ. (Japan); A. Takahashi, Nagaoka National College of Technology (Japan); D. Wakatsuki, Tsukuba Univ. of Technology (Japan)

$743205 \quad$ New calibration technique for a novel stereo camera [7432-04]

X. Tu, M. Subbarao, SUNY, Stony Brook (United States)

743206 3D cutting tool inspection system and its key technologies [7432-05]

X. M. Du, T. Chen, X. J. Zou, GE Global Research (China); K. G. Harding, GE Global Research (United States)

743207 3D measurement method based on wavelet transform by using SEM [7432-06]

Y. Arai, M. Ando, Kansai Univ. (Japan); S. Yokozeki, Jyouko Applied Optics Lab. (Japan)

\section{CALIBRATION AND ANALYSIS METHODS II: DATA ANALYSIS}

743208 A fringe period unwrapping technique for digital fringe profilometry based on spatial shift estimation [7432-07]

P. Cao, J. Xi, J. F. Chicharo, Y. Yu, Univ. of Wollongong (Australia)

743209 Shift-variant image deblurring for machine vision: one-dimensional blur [7432-08] M. Subbarao, Y. Kang, X. Tu, SUNY, Stony Brook (United States)

7432 OA Automatic inspection of textured surfaces by support vector machines [7432-09] S. Jahanbin, A. C. Bovik, The Univ. of Texas at Austin (United States); E. Pérez, D. Nair, National Instruments Corp. (United States)

7432 OB Stokes parameters of reflected and scattered light by a rough surface [7432-10] L. Jin, Univ. of Yamanashi (Japan); K. Takizawa, Seikei Univ. (Japan) 
7432 OC Data processing and parameter extraction for cutting tool inspection [7432-11]

T. Chen, X. M. Du, J. M. Zheng, GE Global Research (China); K. G. Harding, GE Global

Research (United States)

\section{OPTICAL METROLOGY AND 3D APPLICATIONS I}

7432 OD In-process inspection of internal threads of machined automotive parts [7432-12]

H. Zhang, R. Katz, Univ. of Michigan (United States); J. S. Agapiou, General Motors Corp.

(United States)

7432 OE Measure of roughness of paper using speckle [7432-13]

A. Pino, J. Pladellorens, Politècnic Univ. de Catalunya (Spain)

7432 OF A novel method for overlay measurement by scatterometry [7432-14]

W.-T. Hsu, Y.-S. Ku, D.-M. Shyu, Industrial Technology Research Institute (Taiwan)

\section{OPTICAL METROLOGY AND 3D APPLICATIONS II}

$7432 \mathrm{OH}$ In situ analysis of fruit anthocyanins by means of total internal reflectance, continuous wave and time-resolved spectroscopy [7432-16]

M. Zude, Leibniz Institute for Agricultural Engineering Potsdam-Bornim (Germany); L. Spinelli, Politecnico di Milano (Italy); C. Dosche, Univ. of Potsdam (Germany); A. Torricelli, Instituto di Fotonica e nanotecnologie, IFN-CNR, Politecnico di Milano (Italy)

7432 0J Inner surface profile measurement of a hydrodynamic bearing by an oblique incidence and two-wavelength interferometer [7432-18]

O. Sasaki, R. Yamamura, K. Yokoyama, T. Suzuki, Niigata Univ. (Japan)

7432 OK Digital processing of an interferometric velocimeter for ballistic shock measurement [7432-19]

P. Kumar, A. Thomas, R. S. Weis, T. J. Tayag, Texas Christian Univ. (United States)

$7432 \mathrm{OL}$ In-plane and out-of-plane deformation and vibration measurement using an optomechanical image derotator [7432-20]

M. Rahlves, S. Mirzaei, T. Fahlbusch, E. Reithmeier, Leibniz Univ. Hannover (Germany)

\section{D METHODS I: STRUCTURED LIGHT AND PHASE METHODS}

7432 ON Digital multiple wavelength phase shifting algorithm [7432-22]

S. Zhang, lowa State Univ. (United States)

743200 Continuous scanning phase measurement for high immunity to vibration [7432-23] J. Park, J. You, S.-W. Kim, Korea Advanced Institute of Science and Technology (Korea, Republic of)

7432 OP Three-dimensional profilometry system incorporating a MEMS scanner [7432-24]

T. Yoshizawa, T. Wakayama, Saitama Medical Univ. (Japan) 
7432 OS Comparison of projection means for structured light systems [7432-27]

K. Harding, GE Global Research (United States)

3D METHODS II: SPECKLE, HOLOGRAPHIC, AND DYNAMIC METHODS

7432 OT Microscopic TV sherography for microsystems characterization [7432-28]

U. P. Kumar, N. K. Mohan, M. P. Kothiyal, Indian Institute of Technology Madras (India)

7432 OV 3D inspection microscope using holographic primary objective [7432-30]

T. D. Ditto, DeWitt LLC (United States); J. Knapp, S. Biro, Aspex, Inc. (United States)

7432 OW Dual mode interferometer for measuring dynamic displacement of specular and diffuse components [7432-31]

M. North Morris, T. Horner, M. Naradikian, J. Shiefman, 4D Technology Corp. (United States)

3D METHODS III: OTHER METHODS INCLUDING COLOR, STEREO, AND FOCUS

7432 OX Combined stereovision and phase shifting method: use of a color visibility-modulated fringe pattern [7432-32]

X. Han, P. Huang, Stony Brook Univ. (United States); Z. Deng, L. Xu, Nokia Research Ctr. (China)

7432 OY Pixel synchronous measurement of object shape and colour [7432-33]

J. Siepmann, M. Heinze, P. Kühmstedt, G. Notni, Fraunhofer IOF Jena (Germany)

$74320 Z$ Chromatic confocal spectral interferometry for technical surface characterization [7432-34] W. Lyda, D. Fleischle, T. Haist, W. Osten, Univ. Stuttgart (Germany)

743210 Three-dimensional profilometry based on focus method by projecting LC grating pattern [7432-35]

Y. Otani, F. Kobayashi, Y. Mizutani, Tokyo Univ. of Agriculture and Technology (Japan);

T. Yoshizawa, Saitama Medical Univ. (Japan)

743211 A portable 3D shape measurement system based on the combined stereovision and phase shifting method [7432-36]

X. Han, P. Huang, Stony Brook Univ. (United States); Z. Deng, L. Xu, Nokia Research Ctr. (China)

743212 Real-time 3D part metrology using polarization rotation [7432-37]

G. Abramovich, K. Harding, V. Paruchuru, S. Manickam, C. Nafis, J. Czechowski, GE Global Research (United States); A. Vemury, U.S. Dept. of Homeland Security (United States)

743213 Laser Doppler distance sensors using phase and frequency evaluation [7432-38]

J. W. Czarske, T. Pfister, P. Günther, L. Büttner, Dresden Univ. of Technology (Germany) 
743214 Research of the chromaticity coordinates and color spectrum calibration using tristimulus sensors and eigenspectrum method [7432-39]

O.-Y. Mang, T.-W. Huang, Y.-F. Hsieh, National Central Univ. (Taiwan); Y.-T. Kuob, National Chiao-Ting Univ. (Taiwan) and Jen-Teh Junior College of Medicine (Taiwan)

743216 Iterative estimation of the topography by means of structured light [7432-42]

A. Martínez García, J. A. Rayas-Alvarez, Ctr. de Investigaciones en Óptica A.C. (Mexico); H. J. Puga Soberanes, Instituto Tecnológico de León (Mexico); K. Genovese, Univ. degli Studi della Basilicata (Italy)

743217 Multi-channel liquid crystal cell parameter measurement technique [7432-43]

C.-S. Liu, K.-P. Chuang, Y.-S. Lin, M.-Y. Zhuang, C.-J. Chiang, Industrial Technology Research Institute (Taiwan)

743218 Method to measure frequency change of tunable laser based on Jamin shearing interferometer [7432-44]

L. Wang, L. Liu, J. Sun, Y. Zhou, Z. Luan, D. Liu, Shanghai Institute of Optics and Fine Mechanics (China)

7432 1A Measurement of frequency swept linearly with Fabry-Perot fiber interferometer [7432-46] Z. Luan, N. XU, D. Liu, Y. Zhou, L. Liu, Shanghai Institute of Optics and Fine Mechanics (China)

7432 1B Computation of crack tip elastic strain intensity factor in mode I by electronic speckle pattern interferometry [7432-47]

J. Parra Michel, A. Martínez, J. A. Rayas, Ctr. de Investigaciones en Óptica, A.C. (Mexico)

7432 1C Three-dimensional embedded defect detection and localization in a semi-transparent medium [7432-48]

G. Abramovich, C. Nafis, Y. Williams, K. Harding, E. Tkaczyk, GE Global Research (United States)

Author Index 


\title{
Conference Committee
}

\author{
Symposium Chair
}

James G. Grote, Air Force Research Laboratory (United States)

Symposium Cochair

E. Fred Schubert, Rensselaer Polytechnic Institute (United States)

Program Track Chair

Katherine Creath, Optineering (United States) and College of Optical

Sciences, The University of Arizona (United States)

Conference Chairs

Peisen S. Huang, Stony Brook University (United States)

Toru Yoshizawa, Saitama Medical University (Japan)

Kevin G. Harding, GE Global Research (United States)

Program Committee

Motoharu Fujigaki, Wakayama University (Japan)

Qingying Hu, Akrometrix LLC (United States)

Heinz Hügli, University de Neuchâtel (Switzerland)

Seung-Woo Kim, Korea Advanced Institute of Science and Technology (Korea, Republic of)

Gunther Notni, Fraunhofer-Institut für Angewandte Optik und Feinmechanik (Germany)

Guiju Song, GE Global Research (China)

Muralidhara Subbarao, Stony Brook University (United States)

Toshiyuki Takatsuji, National Institute of Advanced Industrial Science and Technology (Japan)

Jiangtao Xi, University of Wollongong (Australia)

Jian Xu, A*STAR Singapore Institute of Manufacturing Technology

(Singapore)

Shenghua Ye, Tianjin University (China)

Stuart S. Yin, The Pennsylvania State University (United States)

Song Zhang, lowa State University (United States)

Session Chairs

1 Calibration and Analysis Methods I: Calibration

Peisen S. Huang, Stony Brook University (United States) 
2 Calibration and Analysis Methods II: Data Analysis

Song Zhang, lowa State University (United States)

3 Optical Metrology and 3D Applications I

Toru Yoshizawa, Saitama Medical University (Japan)

4 Optical Metrology and 3D Applications II

Peisen S. Huang, Stony Brook University (United States)

5 3D Methods I: Structured Light and Phase Methods

Peter Kuehmstedt, Fraunhofer-Institut für Angewandte Optik und Feinmechanik (Germany)

6 3D Methods II: Speckle, Holographic, and Dynamic Methods Kevin Harding, GE Global Research (United States)

7 3D Methods III: Other Methods Including Color, Stereo, and Focus Seung-Woo Kim, Korea Advanced Institute of Science and Technology (Korea, Republic of) 